\title{
Using Sensor Networks for Pedestrian Detection
}

\author{
Aline Senart, Marcin Karpiński, Maciej Więckowski and Vinny Cahill \\ Distributed Systems Group \\ Department of Computer Science \\ Trinity College Dublin \\ \{first.last\}ecs.tcd.ie
}

\begin{abstract}
Pedestrian safety is a major concern in road transportation as pedestrian/vehicle accidents account for the second largest cause of traffic-related injuries and fatalities worldwide. Considerable work has examined the use of lidar, radar and computer vision for pedestrian detection but existing solutions are costly and work only if pedestrians are in the vehicle's lineof-sight. In this paper, we present a novel technique based on wireless sensor networks that is cheap and enables pedestrian detection beyond the driver's horizon. The detection system is based on the use of "cat's eyes" augmented with embedded processing and communication capabilities that are able to detect pedestrians and forward this information along the road. We describe how such a system was successfully built with SOL, a high-level programming language for wireless sensor networks. Initial results show that the system obtains detection rates of $100 \%$, false positive rates of $0 \%$, and that the precision of the estimated position of pedestrians depends on their heading and relative position to sensor nodes.
\end{abstract}

Index Terms-Pedestrian detection system, wireless sensor networks, cat's eyes, radio.

\section{INTRODUCTION}

An estimated 1.17 million deaths occur each year worldwide due to road accidents [1]. Pedestrian/vehicle accidents are the second largest source of traffic-related injuries and fatalities after accidents involving only car passengers [2]. The majority of these deaths occur in developing countries but the pattern of road-user fatalities is the same in developed countries. 49 pedestrians were killed in road accidents in Ireland in 2007 to the 20th of August out of a total of 216 road users killed [3]. Pedestrian detection is therefore essential to reduce this tragic level of pedestrian injuries and fatalities.

Many interesting approaches for pedestrian detection have been proposed. Such work has examined a range of technologies including lidar, radar and computer vision (using infrared and/or conventional cameras), and has studied their use on their own or in combination with each other [2], [4]. However, imaging sensors using visible light or infrared radiation are relatively expensive and involve a substantial

\footnotetext{
${ }^{0}$ (C) 2008 IEEE. Personal use of this material is permitted. However, permission to reprint/republish this material for advertising or promotional purposes or for creating new collective works for resale or redistribution to servers or lists, or to reuse any copyrighted component of this work in other works must be obtained from the IEEE. This material is presented to ensure timely dissemination of scholarly and technical work. Copyright and all rights therein are retained by authors or by other copyright holders. All persons copying this information are expected to adhere to the terms and constraints invoked by each author's copyright. In most cases, these works may not be reposted without the explicit permission of the copyright holder.
}

amount of processing. Time-of-flight sensors such as radar and lidar are cheaper but they are traditionally mounted on vehicles and therefore have limited visibility [4].

In this paper, we present a novel technique based on a largescale wireless sensor network of "cat's eyes" augmented with cheap embedded processing and communication capabilities. In this pedestrian detection system, reflective armbands or night vision jackets worn by pedestrians are equipped with communication capabilities that send radio signals periodically. These beacons are received by the cat's eyes placed along the road and can be used to infer, thanks to the strength of the signals, the presence and approximate position of pedestrians. This information is forwarded from cat's eye to cat's eye allowing vehicles to be informed about the pedestrians presence beyond their drivers' line of sight.

Our approach of requiring pedestrians to carry tiny, selfcontained devices with radios seems reasonable as wearable computing moves from research laboratories to the real world [5]. For instance, digital avalanche transceivers [6], [7] are today integrated into apparel, helmets, protection gear or boots, and mobile phones are increasingly becoming ubiquitous computational devices [8]. Furthermore, pedestrians often already wear flashing beacons and high-visibility safety garments with retroreflective trim [9].

We describe how such a pedestrian detection system was successfully built using SOL [10], a high-level programming language for wireless sensor/actuator networks that allows programmers to use a fine-grained concurrency model and enables a clear boundary definition between application code and the underlying platform.

Initial results show that the system obtains detection rates of $100 \%$ and false positive rates of $0 \%$. The position estimation has a precision of more than $95 \%$ with a $5 \mathrm{~m}$ accuracy when pedestrians are facing along the road (as opposed to facing the opposite side of the road) and varies depending on their heading and relative position to sensor nodes.

This paper is organised as follows: Section II presents related work in pedestrian detection; Section III introduces the wireless sensor network-based pedestrian detection system; Section IV presents the implementation of this approach and Section V details the performance of the system. Section VI summarises and concludes the paper. 


\section{RELATED WORK}

A variety of vehicle-based technologies have emerged in recent years that offer promising approaches for detecting pedestrians. A general overview of these technologies is given by Gavrilla et al. in [2].

Commonly used sensors for detecting pedestrians are imaging sensors using visible light [11] or infrared radiation [12], [13]. Visible light sensors capture detailed descriptions of a road scene but are not effective during night-time or in bad weather. On the other hand, infrared sensors do not depend on illumination and can be used during the day or at night time with little difference. However, infrared sensors are not effective under high temperature conditions and clothes affect the pedestrians' thermal footprint [14]. Pedestrian detection using imaging sensors is also a high-cost solution for two reasons. The first reason stems from the need for vision algorithms that are computationally intensive and require stateof-the art CPUs. The second reason is that several imaging sensors are traditionally used simultaneously to obtain depth information, and both vision and thermal cameras are often combined to exploit the advantages of both approaches [14].

Other approaches mount time-of-flight sensors such as radar [15] and lidar [16], [17] on vehicles since they are significantly cheaper and measure distances directly. Radar emits electromagnetic waves, which are reflected by the target and detected by a receiver that is able to identify the range, direction, or speed of the target. To distinguish pedestrians from other potential targets, the power spectral-density plot of the reflected signals are examined [2]. Instead of using radio waves like radar technology, lidar uses light pulses (e.g., laser) and determines the range of a distant target by measuring the time delay between transmission of pulses and detection of the reflected signals. Both radar and lidar systems suffer from interference. They must overcome sources of unwanted signals and have to discern the desired signals from background noise in order to focus only on the actual targets of interest.

To cope with the deficiencies of each approach, previous work has studied the combination of multiple sensor modalities [14], [18], [19]. Even though improving on the results obtained from a single sensor system, they still provide only limited visibility since only line-of-sight sensors mounted on vehicles are used [4]. The area in which pedestrians can be detected is restricted to the surroundings of the vehicles. Hence it is useful to complement these vehicle-based approaches with sensors deployed in the infrastructure.

Infrastructure-mounted cameras are extensively used for video surveillance [20] but are only placed at busy or dangerous intersections, near schools and on blind curves due to their high cost. An approach based on sensor networks therefore seems to be a good alternative as typical applications of sensor networks include large-scale monitoring and tracking at very low cost. Astucia manufactures devices called "road studs" [21] that are placed in the road surface and equipped with light, humidity and temperature sensors, and also with a number of bright LEDs that change colour depending on

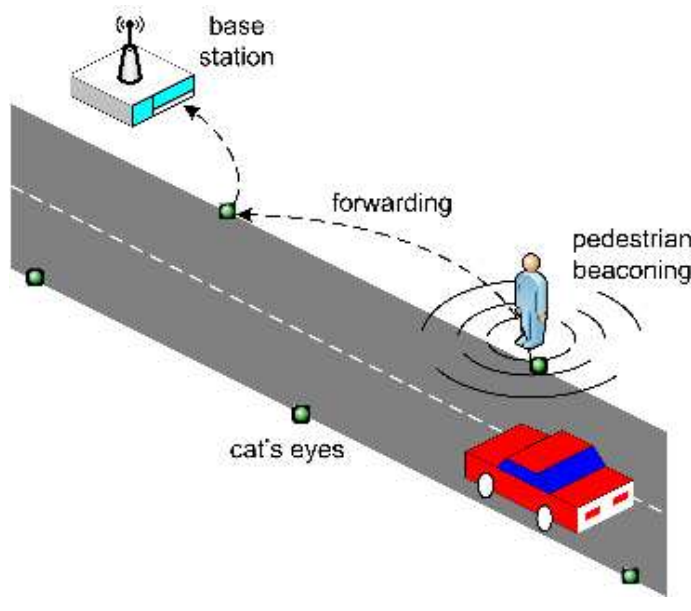

Figure 1. Pedestrian detection system

weather conditions. However, the studs do not interact with each other and can therefore not be considered as part of a sensor network. As far as we know, no work on sensor networks for pedestrian detection has yet been published.

\section{Modeling the Pedestrian Detection System}

According to the Irish National Roads Authority, $72 \%$ of all road accidents occur outside of urban environments. Almost $80 \%$ of all road accidents in Ireland take place on two-way single carriageways, which makes this the most dangerous type of road [22]. Given these numbers, we consider two-way single carriageways in rural environments as the primary target deployment environment for our pedestrian detection system, although our approach is applicable to any type of road.

In our approach to increasing road safety in rural environments, cheap sensor nodes are deployed inside cat's eyes over long distances along both lanes of the carriageway (see Fig. 1). The nodes are equipped with minimal processing and communication capabilities (e.g., radio). After deployment, they form a large-scale wireless sensor network that collaboratively computes the positions of pedestrians walking (or running) along the road.

To be detected, pedestrians have to wear reflective armbands or night vision jackets that are equipped with communication capabilities. These high-visibility safety garments send radio beacons that are received by one or more of the sensor nodes. Thanks to the measurement of the received radio signal strength (RSSI), the presence and position of the pedestrians can be inferred (see Section IV).

Information about pedestrians is forwarded between the sensor nodes to a roadside base station that determines in real time the movements of the pedestrians. The base station may display warnings through variable-message signs (VMS), report to a control centre or, in the future, wait for a vehicle to come in range to send information about the pedestrians to its on-board computer. This provides drivers with a consistent view of the road situation beyond their horizon (i.e., a hundred meters ahead of them), so they can react to the presence of 


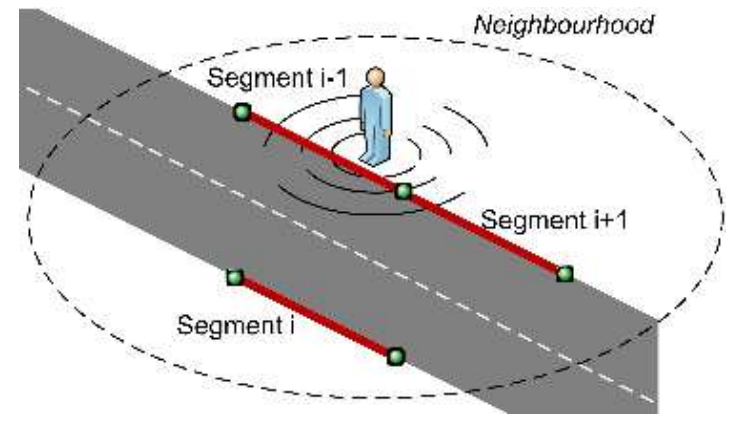

Figure 2. Segments between sensor nodes

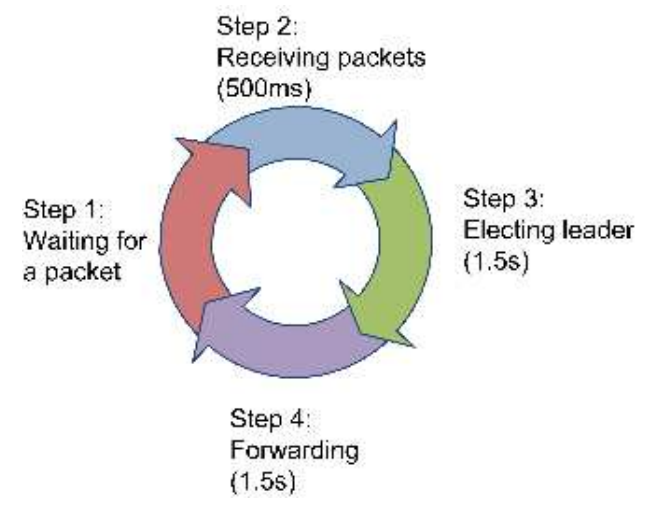

Figure 3. Algorithm timeline

pedestrians early enough.

The most critical task in sensor networks is energy saving [23]. Minimising communication and employing wakeup schemes that turn off sensor nodes when possible are effective in energy saving, but are out of scope of this paper.

\section{Implementing the Pedestrian Detection SYSTEM}

To implement the pedestrian detection system, we used SOL, a programming language for wireless sensor/actuator networks that raises the level of abstraction of TinyOS and thus makes sensor/actuator network applications easier to program, while maintaining good performance [10]. The NesC code [24] produced by the SOL compiler is fully functional and can be compiled immediately with the NesC compiler and deployed on the motes.

At deployment time, each sensor node is given the identity of its immediate and opposite neighbours, allowing the definition of segments, that are possible positions of the pedestrians in its neighbourghood (see Fig. 2). The algorithm that is executed after initialisation by each sensor node is the following (see Fig. 3):

- First, sensor nodes wait for a packet from a pedestrian.

- When a sensor node receives such a packet, it waits for 3 other packets (a beacon contains 4 packets). Packets that are missing after $500 \mathrm{~ms}$ are assigned a RSSI value ${ }^{1}$

${ }^{1}$ As reported by the TinyOS 2.x CC1000 radio stack.

\begin{tabular}{|l|l|l|l|l|l|l|}
\hline Sender Id & Beacon Nb & Local RSSI & RSSI A & RSSI B & Node A & Node B \\
\hline
\end{tabular}

Figure 4. Structure of the messages exchanged between sensor nodes

\begin{tabular}{|l|l|l|l|l|l|l|}
\hline Sender Id & Origin Id & Beacon Nb & RSSI A & RSSI B & Node A & Node B \\
\hline
\end{tabular}

Figure 5. Structure of the messages forwarded to the base station

of 400. This accounts from the fact that the absence of packet should not be treated as if a packet was received since it gives an indication on the detection accuracy. The value of 400 is sufficiently high to significantly lower the average RSSI calculated from the 4 packets, thus allowing comparisons of RSSI values between sensor nodes that have and have not received 4 packets.

- Then, the sensor node broadcasts a "detected" message to its neighbours and stores all the messages that it receives from them. As depicted in Fig. 4, the messages contain the identity of the sender, the number and average RSSI values of the received beacons and the current knowledge that the sender has about the segment where the best RSSI value has been detected (i.e., where the pedestrian is located), including the identity of the nodes at the ends of the segment and their RSSI values. Several rounds of messages are exchanged until either a timeout of $1.5 \mathrm{~s}$ expires or the segment with the highest RSSI value is elected (i.e., when neighbouring sensor nodes reach a consensus on the identity of the segment).

- Finally, the sensor node located on one end of the elected segment (or the segments with the highest RSSI value in case of a timeout) that received the highest RSSI value forwards information about the presence of the pedestrian to the sensor nodes along the road to report to the base station. This forwarding activity is limited to 1s. As depicted in Fig. 5, the forwarded messages contain the identity of the sender, the origin of the beacon (i.e., the node that received the highest RSSI), the beacon number and the position of the pedestrian in terms of the RSSI values and identities of the nodes at the ends of the elected segment.

The time bound that we used at each step of the algorithm ensures the responsiveness of the system. Communication and processing activities between the reception of the first packet and the report to the base station last exactly $3.5 \mathrm{~s}$, that is less than the period of the pedestrians' beacons, ensuring that the sensor nodes are fully operational when a beacon is received.

\section{Evaluation}

The system that we built for our experiments consists of a Dell D400 running Ubuntu 7.04 acting as a base station and 16 Mica2 motes [25] running TinyOS 2.0.2 and deployed on both sides of a $6 \mathrm{~m}$-wide road, covering a total distance of $75 \mathrm{~m}$ (see Fig. 6). The distance between the motes was $10 \mathrm{~m}$. 


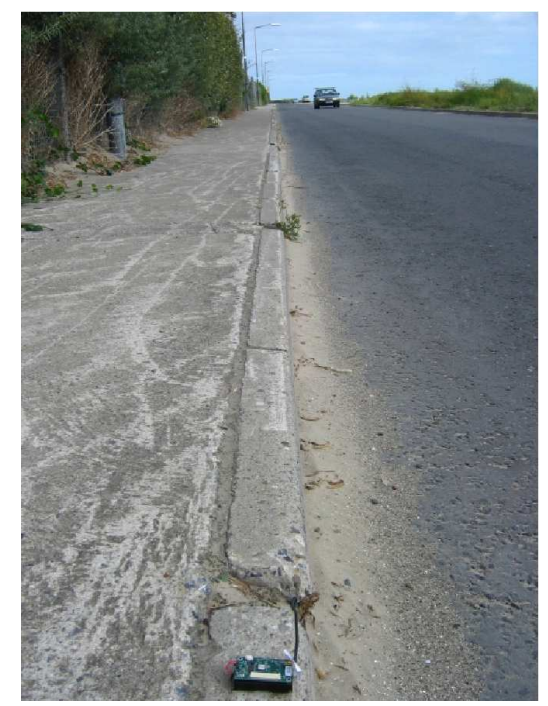

Figure 6. Deployment on a two-way single carriageway

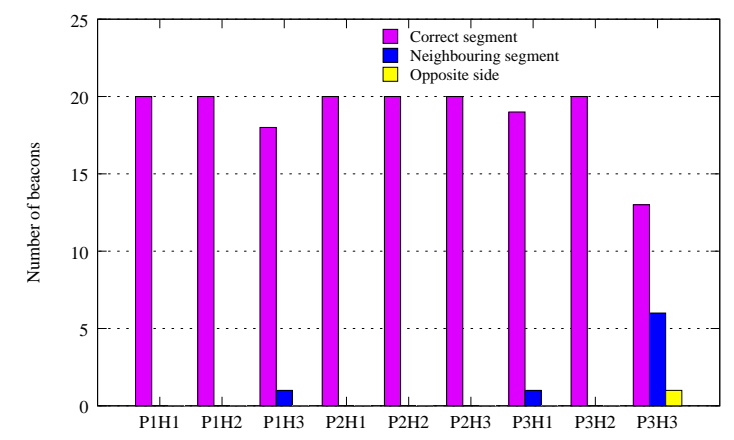

Figure 7. Position estimation vs. position and heading of pedestrians

Each pedestrian walking along the path was outfitted with a beaconing mote whose period was $4 \mathrm{~s}$.

We carried out two sets of experiments: (i) detection of static pedestrians and (ii) detection of moving pedestrians. In both cases, results showed that the detection rate of the system is $100 \%$ and the false positive rate is $0 \%$, i.e., all pedestrians were detected.

In the first set of experiments, we additionally wanted to evaluate if the position and the direction of the pedestrians within a segment would have an impact on the position detection rate. We did extensive experiments and noticed that our results were symmetric for both sides of the road and for the different segments. We present in Fig. 7 the results that we obtained for 20 beacons emitted by a static pedestrian with 3 different positions and 3 different headings, as illustrated in Fig. 8. $\mathrm{P} i \mathrm{H} j$ corresponds to position $i$ with heading $j$, with $i, j \in 1 . .3$, where $\mathrm{P} 1$ corresponds to the position close to a sensor node, P3 is located between two sensor nodes, P2 is between $\mathrm{P} 1$ and $\mathrm{P} 3, \mathrm{H} 1$ is perpendicular to the road, $\mathrm{H} 3$ is facing along the road and $\mathrm{H} 2$ is between $\mathrm{H} 1$ and $\mathrm{H} 3$.

These results show that most of the time pedestrians are detected in the correct segment. To improve the $10 \mathrm{~m}$ precision that can be obtained with this segment approach, we designed

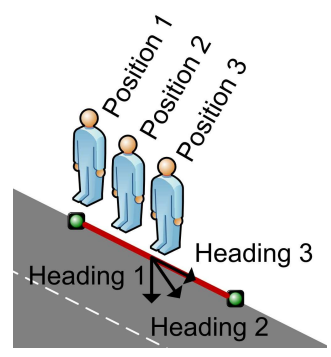

Figure 8. Positions and headings used for the evaluation

\begin{tabular}{|c||c|c|c|}
\hline & Position1 & Position2 & Position3 \\
\hline \hline Heading1 & $100 \%$ & $45 \%$ & $60 \%$ \\
\hline Heading2 & $100 \%$ & $100 \%$ & $95 \%$ \\
\hline Heading3 & $95 \%$ & $100 \%$ & $100 \%$ \\
\hline
\end{tabular}

Table I

PERCENTAGE OF CORRECT POSITION ESTIMATIONS WITH THE IMPROVED METHOD

a novel method. In this method, we estimate the position of a pedestrian within the elected segment according to the RSSI values of the sensor nodes located at its ends. If the difference between their values is lower than a threshold, then we can infer that the position of the pedestrian is approximately in the middle of the segment. However, if the difference exceeds the threshold, then the pedestrian is more likely to be close to the sensor node that has a better RSSI reading. After multiple experiments, we determined that the optimal threshold for our settings was a RSSI value of 70 .

With this method, we increase the granularity of position estimation so that, instead of predicting just the section in which the pedestrian is, we predict in which of the $5 \mathrm{~m}$-wide regions within this section it is (i.e., the $5 \mathrm{~m}$-wide regions centered around each sensor node and around the middle point of each section). This allows us to achieve an average accuracy of $5 \mathrm{~m}$ and this result can be refined down to $2.5 \mathrm{~m}$ since whenever the system answers that a pedestrian is located between nodes $\mathrm{A}$ and $\mathrm{B}$ with an indication of being closer to $A$, it effectively means that the pedestrian must be in a region that starts at $\mathrm{A}$ and extends $2.5 \mathrm{~m}$ towards $\mathrm{B}$. To evaluate the improved accuracy method, we applied it to the results obtained previously but this time taking into account only the cases when the system correctly predicted on which side of the road the pedestrian was. The percentage of correct position estimations is presented in Table I. For these results, the incorrect position estimations correspond to the maximum of $6.5 \mathrm{~m}$ and $7.5 \mathrm{~m}$ estimation error for Position 2 and Position 3 respectively with Heading1. This low percentage of detection for Heading 1 can be explained easily: when the pedestrian is facing the opposite side of the road, the body of the pedestrian prevents the segment in which it is actually located properly receiving the beacons.

In the second set of experiments, we wanted to evaluate the position detection rate of pedestrians walking. Fig. 9 presents results obtained for two walks, one on the left-hand 


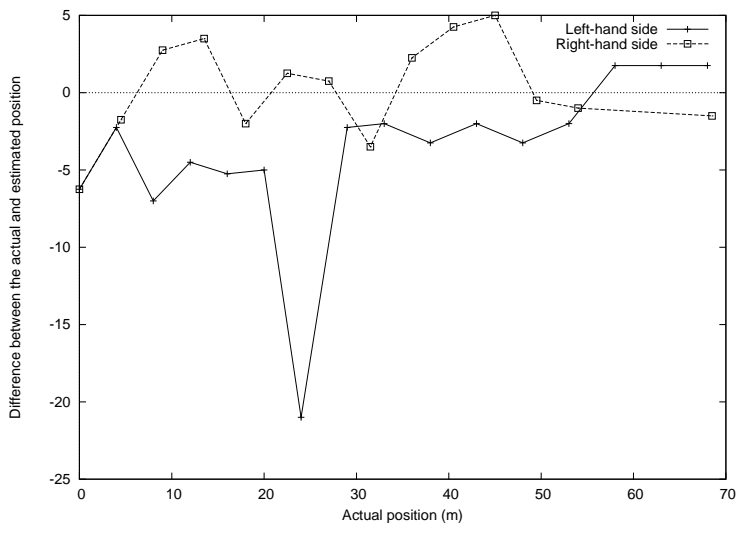

Figure 9. Difference between the actual and estimated position of a walking pedestrian

side and one on the right-hand side of the road with an average walking speed of $1.12 \mathrm{~m} / \mathrm{s}$. As shown in the figure, the difference between the actual and estimated position is small (around 5m), except for the beacon 7 of the first walk. This variation is due to the movements of the pedestrian that change the direction of the antenna. In some situations, the antenna can be oriented towards the opposite side of the road in which case, we obtain results similar to those obtained for Heading1 with the static pedestrians. Also, on average, $24 \%$ of the predictions were detected on the wrong side of the road. To tackle problems of this sort, more advanced probabilistic methods based on previous positions could be used.

Given these experiments, we note that detecting the position of pedestrians is not deterministic. The orientation of the antenna that the pedestrians carry makes a significant difference in signal propagation. Better results are obtained with a horizontal antenna directed towards the road. In order to evaluate the influence of environmental factors (like fences along the road and cars passing), it would be interesting to perform exactly the same experiments in open spaces and use the results as a point of reference. Further experiments could also evaluate the impact of walking speed, weather, distance between the sensor nodes, width of the road, etc. but we feel that they will have only a slight influence on the results.

\section{CONCLUSION}

In this paper, we have presented a pedestrian detection system that makes road transportation safer by detecting $100 \%$ of pedestrians along roads without any false detection. The presence of the pedestrians is inferred by cat's eyes augmented with embedded processing and communication capabilities that are able to detect beaconing pedestrians and calculate their position with a precision of more than $95 \%$ when pedestrians are oriented toward the road. The information is forwarded from cat's eye to cat's eye to a roadside base station that is responsible to inform incoming drivers. Even though the approach is simple and cheap, results are promising and pedestrian detection is performed beyond driver's horizon. Future work will include devising security protocols to protect the system against illicit tracking of pedestrians and potential attacks.

\section{REFERENCES}

[1] "Roads \& highways: Road safety," The World Bank Group, http://www.worldbank.org.

[2] D. M. Gavrilla, "Sensor-based pedestrian protection," IEEE Intelligent Systems, vol. 16, no. 6, pp. 77-81, 2001 .

[3] "Provisional fatal collision statistics 2007," http://www.garda.ie, August 2007, Ireland.

[4] T. Gandhi and M. M. Trivedi, "Pedestrian collision avoidance systems: A survey of computer vision based recent studies," in 9th International IEEE Conference on Transportation Systems, Toronto, Canada, September 2006.

[5] R. Hull, J. Reid, and E. Geelhoed, "Creating experiences with wearable computing," IEEE Pervasive Computing, vol. 1, no. 4, pp. 56-61, OctDec 2002.

[6] F. Michahelles, P. Matter, A. Schmidt, and B. Schiele, "Applying wearable sensors to avalanche rescue: First experiences with a novel wearable avalanche beacon," in International Workshop on Mobile Computing Assistance, Mobility, Applications, Rostock, Germany, June 2003.

[7] J. J. Modroo and G. R. Olhoeft, "Avalanche rescue using ground penetrating radar," in Tenth International Conference on Ground Penetrating Radar, Delft, The Netherlands, June 2004, pp. 785-788.

[8] C. Roduner, M. Langheinrich, C. Floerkemeier, and B. Schwarzentrub, "Operating appliances with mobile phones - strengths and limits of a universal interaction device," in Fifth International Conference on Pervasive Computing, Toronto, Canada, May 2007.

[9] J. R. Sayer and M. L. Mefford, "The roles of garment design and scene complexity in the daytime conspicuity of high-visibility safety apparel," University of Michigan Transportation Research Institute, Tech. Rep. UMTRI-2005-5, February 2005, Industry Affiliation Program for Human Factors in Transportation Safety.

[10] M. Karpiński and V. Cahill, "High-level application development is realistic for wireless sensor-actuator networks," in Fourth Annual IEEE Communications Society Conference on Sensor, Mesh and Ad Hoc Communications and Networks, San Diego, California, USA, June 2007.

[11] D. M. Gavrilla and S. Munder, "Multi-cue pedestrian detection and tracking from a moving vehicle," International Journal of Computer Vision, vol. 73, no. 1, pp. 41-59, 2007.

[12] M. M. D. Linzmeier, J. Dickmann, and K. C. J. Dietmayer, "Pedestrian detection with thermopiles using an occupancy grid," in IEEE Intelligent Transportation Systems Conference, Washington, D.C., USA, October 2004, pp. 1063-1068.

[13] M. Bertozzi, A. Broggi, C. Caraffi, M. D. Rose, M. Felisa, and G. Vezzoni, "Pedestrian detection by means of far-infrared stereo vision," Computer Vision and Image Understanding Journal, vol. 106, no. 23, pp. 194-204, May 2007, Elsevier.

[14] M. Bertozzi, A. Broggi, M. Felisa, G. Vezzoni, and M. D. Rose, "Lowlevel pedestrian detection by means of visible and far infra-red tetravision," in IEEE Intelligent Vehicles Symposium, Tokyo, Japan, June 2006.

[15] M. Klotz and H. Rohling, "24 GHz radar sensors for automotive applications," in 13th International Conference on Microwaves, Radar and Wireless Communications, vol. 1, Wroclaw, Poland, 1998, pp. 359362.

[16] A. Fod, A. Howard, and M. J. Mataric, "Laser-based people tracking," in IEEE International Conference on Robotics and Automation, Washington, D.C., USA, May 2002.

[17] K. C. Fuerstenberg and K. Dietmayer, "Pedestrian detection and classification by laserscanners," in IEEE Intelligent Vehicles Symposium, Parma, Italy, June 2004.

[18] U. Scheunert, H. Cramer, B. Fardi, and G. Wanielik, "Multi sensor based tracking of pedestrians: A survey of suitable movement models," in IEEE Intelligent Vehicles Symposium, Parma, Italy, June 2004.

[19] S. Milch and M. Behrens, "Pedestrian detection with radar and computer vision," Smart Microwave Sensors, 2007, http://www.smart-microwavesensors.de/Pedestrian_Detection.pdf.

[20] L. Hempel and E. Topfer, "CCTV in Europe," http://www.urbaneye.net, August 2004.

[21] "Solarlite intelligent road studs," Astucia Traffic Safety Systems, 2007, http://www.astucia.co.uk.

[22] "Road collisions facts," National Road Authority, 2004, Ireland. 
[23] S. J. Baek, G. de Veciana, and X. Su, "Minimizing energy consumption in large-scale sensor networks through distributed data compression and hierarchical aggregation," IEEE Journal on Selected Areas in Communications, vol. 22, no. 6, pp. 1130-1140, August 2004.

[24] D. Gay, P. Levis, R. von Behren, M. Welsh, E. Brewer, and D. Culler, "The NesC language: A holistic approach to networked embedded systems," in ACM SIGPLAN Conference on Programming Language Design and Implementation, San Diego, California, USA, 2003, pp. 1 11.

[25] "Mica2 868, 916 MHz," Crossbow Wireless Sensor Networks, 2007, http://www.xbow.com/Products/productdetails.aspx?sid=174. 